\title{
A Novel Method for Investigating Finger-based Representations using Virtual Reality
}

\author{
Alyson M. de C. Souza \\ alyson@imd.ufrn.br \\ Federal University of Rio Grande do Norte \\ Natal, RN, Brazil \\ Leibniz-Institut für Wissensmedien \\ Tübingen, Germany \\ Korbinian Moeller \\ K.Moeller@lboro.ac.uk \\ Loughborough University \\ Leicestershire, UK \\ Leibniz-Institut für Wissensmedien \\ Tübingen, Germany
}

\author{
Roberta Barrocas \\ r.barrocas@iwm-tuebingen.de \\ Leibniz-Institut für Wissensmedien \\ Tübingen, Germany
}

César Rennó-Costa

cesar@imd.ufrn.br

Federal University of Rio Grande do Norte

Natal, RN, Brazil

\begin{abstract}
Finger use plays an important role in the development of cognitive (e.g., numerical) concepts. Such embodied relationship between fingers and numbers persists even into adulthood when fingers are typically no longer used for counting. Better understanding how this embodied relationship builds up and plays out is a relevant and ongoing endeavor. To help researchers on this subject, we propose a novel method to investigate finger-based representations using virtual reality. The system is based on virtual hands that match the participant's real hands through tracking and allows for manipulating the virtual model to produce incoherencies between the actual physical finger pattern and the virtual one. We briefly introduce the system components and discuss the first results of the usability of the system.
\end{abstract}

\section{KEYWORDS}

virtual reality, numerical cognition, virtual environments, finger counting, embodied cognition, cognitive science.

\section{INTRODUCTION}

Using fingers is often one of the first strategies used by children for dealing with numbers. This was observed to influence (the development of) number processing in different ways, even in adults [6]. Such influence appears to be stronger when specific patterns of fingers are used to represent numbers - so-called canonical finger patterns (i.e., culturally prevalent finger postures for depicting numerical quantities such as index and middle finger representing 2 in Brazil). For example, numerical magnitudes are recognized faster when displayed in canonical as opposed to non-canonical patterns [5], suggesting that embodied representations of numbers influence number processing.

Two main lines of behavioral research have explored signatures of the embodiment of fingers in adult number processing. The first one investigates sensorimotor influences on adult number processing (e.g., [2]), whereas the second one pursues the question of how visual recognition of specific finger numeral patterns grants access to mental representations of numbers (e.g., $[3,5])$, and how individual finger counting habits modulates this effect. While both lines substantiate that finger patterns activate numerical representations, there is still a lot to learn about the embodiment itself that is involved in the phenomena. Virtual reality (VR) emerges as a relevant technology that can be used to manipulate ones' sense of embodiment in virtual environments and try to provide new insights into how this influences number processing. The goal of this work is to create a new model for embodied cognition researchers to use, that is easy to build and has enough documentation available, providing an innovative way to address embodied cognition research that was not possible before. We aim to develop and validate a custom-made device for finger stimulation and its integrated virtual environment to be used in this context.

The SVR topic for this work is Perception presence, and cognition in $V R / A R / M R$ and this is an ongoing Ph.D. thesis.

\subsection{Motivation}

Virtual Reality is being used by cognitive and neuroscientists to broaden possibilities for experimentation [4]. In embodied cognition research regarding finger-based numerical representations, VR can be used to detach visual and motor inputs received by a participant while producing a specific finger pattern with their own hand. This allows researchers to disentangle each of those perceptual systems' influence on the cognitive experience. This is relevant because experimentally manipulating experienced levels of embodiment over one's own hand is not trivial using other techniques. VR, on the other hand, can use known manipulations of presence and immersion [9] to trigger a sense of ownership over a virtual hand and then manipulate it to generate naturally impossible scenarios.

\subsection{Related Work}

Although different approaches have been taken to study finger movements' influence on number processing, VR has never been used to achieve the decoupling of physical and visually perceived finger postures. Studies such as [7] found that the perception of closing or opening hand movements can lead to a bias towards 
generating smaller or larger numbers, respectively . Interestingly, this effect was only observed for human-looking hands, but not for robot-like hands [1]. Nevertheless, no study to date explored the effect of observing one's own hands on numerical cognition, as done in the present study.

Another line of work focuses on the influence of tactile stimulation on number processing. For example, [8] used a braille cell to stimulate specific finger patterns. However, these studies only shed light on the motor perceptual features of (embodied) number processing. The present work seeks to provide means for simultaneous visual and tactile stimulations, granting the possibility of dissociating them.

\section{PROPOSED SOLUTION}

This work aims to provide a complete solution that allows the use of VR to approach the respective research questions. To achieve this, we developed a VR system for the Oculus Quest that uses the built-in hand tracking embedded in the hardware to show VR hands to a participant and then manipulate them. The system allows the decoupling of the participant's physical finger postures from what they actually see in the virtual environment without breaking immersion. We achieved this by swapping one or more of the fingers moved by the participant with another ones on the VR hand, so that the virtual hand still appeared to move naturally but displayed a finger pattern different from the one currently produced by the participant. This is the central point of the system, as it allows us to manipulate the congruence of motor and visual inputs in ways that were neither thoroughly explored nor possible before. For instance, the participant may lift his index and middle finger (i.e., using his motor system to create the canonical pattern for 2) but see his VR hand stretching index finger and pinky, constituting a non-canonical finger pattern.

Besides, the system can also convey more classical experimental setups, allowing to evaluate the efficacy of VR as a tool compared to many behavioral methods that have been tested before. For this end, the system shows the stimuli in a virtual blackboard (e.g., photos of produced finger patterns and Arabic numbers for comparison with physically produced or virtually seen finger patterns), reflecting standard experiments in numerical cognition research.

Lastly, to complete the experimental setup, we also created a piece of hardware capable of physically stimulating the participant's fingers to elicit numerical patterns without using verbal or visual stimulation, which might otherwise interfere with experimental results. The equipment uses an Arduino board and five vibration motors attached to the participant's fingertips, allowing us to stimulate fingers individually according to the pattern the participant is meant to experience and reproduce .

The system reads from a list of stimuli and presents each trial following those pre-specified experimental conditions. During each trial, the system records the onset time of the presented motor and visual stimuli and the participant's response times for later analysis.

\section{PRELIMINARY RESULTS}

The system has been tested for its usability and viability. The fingerstimulating hardware went through different stress tests that validated that it can deliver stimulation without delays and for a long time without interruptions or failures. The motors were also tested for reliability and were observed to stimulate the respective fingers adequately. The VR system was tested with different hand patterns (both canonical and non-canonical) and all possible conversions. It could create adequate responses for all the cases, except for patterns exclusively involving pinky and thumb.

\section{CONCLUSIONS}

In this article, we presented a novel method for investigating fingerbased representations using virtual reality. In particular, the presented system allows for the independent manipulation of tactile and visual stimulation, allowing experimentally induced incoherencies between these two perceptual channels. Beyond investigating embodied influences on cognitive (number) processing, this method may also allow the evaluation of influences of embodiment experiences on how participants interact with VR and how they perceive their body parts and concepts related to them.

\section{ACKNOWLEDGEMENTS}

This study was financed in part by the Coordenação de Aperfeiçoamento de Pessoal de Nível Superior - Brasil (Capes) - Finance Code 001 .

\section{REFERENCES}

[1] Arnaud Badets, Cédric A Bouquet, François Ric, and Mauro Pesenti. 2012. Number generation bias after action observation. Experimental brain research 221, 1 (2012), 43-49.

[2] Arnaud Badets, Mauro Pesenti, and Etienne Olivier. 2010. Response-effect compatibility of finger-numeral configurations in arithmetical context. Quarterly fournal of Experimental Psychology 63, 1 (2010), 16-22.

[3] Roberta Barrocas, Stephanie Roesch, Verena Dresen, Korbinian Moeller, and Silvia Pixner. 2020. Embodied numerical representations and their association with multi-digit arithmetic performance. Cognitive processing 21, 1 (2020), 95-103.

[4] Jim Blascovich, Jack Loomis, Andrew C Beall, Kimberly R Swinth, Crystal L Hoyt, and Jeremy $\mathrm{N}$ Bailenson. 2002. Immersive virtual environment technology as a methodological tool for social psychology. Psychological inquiry 13, 2 (2002), 103-124.

[5] Samuel Di Luca and Mauro Pesenti. 2008. Masked priming effect with canonical finger numeral configurations. Experimental Brain Research 185, 1 (2008), 27-39.

[6] Frank Domahs, Korbinian Moeller, Stefan Huber, Klaus Willmes, and HansChristoph Nuerk. 2010. Embodied numerosity: implicit hand-based representations influence symbolic number processing across cultures. Cognition 116, 2 (2010), 251-266.

[7] Stéphane Grade, Arnaud Badets, and Mauro Pesenti. 2016. Influence of finger and mouth action observation on random number generation: an instance of embodied cognition for abstract concepts. Psychol. Res 81 (2016), 538-548.

[8] Elena Sixtus, Oliver Lindemann, and Martin H Fischer. 2020. Stimulating numbers: signatures of finger counting in numerosity processing. Psychological research 84 , $1(2020), 152-167$

[9] Mel Slater, Beau Lotto, Maria Marta Arnold, and María Victoria Sánchez-Vives. 2009. How we experience immersive virtual environments: the concept of presence and its measurement. Anuario de Psicología, 2009, vol. 40, p. 193-210 (2009). 